\title{
La mélancolie entre le cerveau et les circonstances: Cabanis et la nouvelle science de l'homme
}

Mariana Saad

\section{Summary}

The period under study ends with the publication of a major work, Rapports du physique et du moral de l'homme, in which Cabanis defends the radical thesis of the identity of the physical and the moral. Amongst all the illnesses studied in this work, he accords particular importance to melancholy as it provides the best way of observing the "physical artifice of thought". Cabanis's rereading of the theses of classical medicine, which is here based on certain carefully chosen studies of the anatomists of his time, enables him to understand melancholy as both a moral affection (great sadness, exalted imagination) and a physical malfunction affecting the organs (namely the brain and the lower abdomen). He is thus able to demonstrate that it can be cured.

Keywords: Pierre Jean Georges Cabanis (1757-1808); brain; melancholy; materialism; sensibility

\section{Résumé}

La période étudiée se clôt avec la parution d'une œuvre majeure, les Rapports du physique et du moral de l'homme. Son auteur, Pierre Jean Georges Cabanis, y défend la thèse radicale de l'identité du physique et du moral.Dans cet ouvrage, parmi toutes les affections qu'il examine, il accorde une importance toute particulière à la mélancolie, car elle permet mieux qu'aucune autre d'observer «l'artifice physique de la pensée». Cabanis propose une relecture des thèses de la médecine antique et s'appuie sur certaines découvertes soigneusement choisies des médecins anatomistes de son temps pour

Dr Mariana Saad (Visiting Research Fellow, History Department, University of Sussex), 29 Shaa Road, London W3 7LW, UK (mariana_saad@hotmail.com). 
penser la mélancolie à la fois comme une affection du moral (la très grande tristesse, l'imagination exaltée) et comme un dysfonctionnement physique, atteignant les organes (en l'occurrence, le cerveau et le bas-ventre). Ceci lui permet d'en démontrer la curabilité.

La pensée médicale connaît en France, dans les dernières années du XVIII ${ }^{\mathrm{e}}$ siècle, un bouleversement majeur avec la publication de la première partie des Rapports du physique et du moral de l'homme ${ }^{1}$ de Pierre Jean Georges Cabanis. Une véritable rupture avec la tradition s'opère alors qui doit entrâ̂ner d'importants changements dans la manière de comprendre la mélancolie. Dans son ouvrage, Cabanis propose en effet d'étudier l'homme à partir d'une donnée radicale: «Le physique et le moral se confondent à leur source; ou, pour mieux dire, le moral n'est que le physique, considéré sous certains points de vue particuliers.» ${ }^{2}$ La portée polémique de cette proposition est toute entière dans la formule restrictive «le moral n'est que le physique». Les Rapports détruisent toute idée d'une supériorité du moral sur le physique, d'une hiérarchie qui placerait au-dessus de la réalité matérielle, l'âme spirituelle.

Dans ce travail de refondation du savoir médical, la mélancolie occupe une place fondamentale en raison du statut qui est traditionnellement le sien de «maladie de l'âme». Ainsi, dès le premier mémoire, de portée générale, «sur l'étude de l'homme», Cabanis annonce un renversement sans précédent dans l'histoire de l'analyse de cette affection. Il se pose en effet la question, tout à fait étonnante pour la tradition, des «organes particuliers du sentiment» ${ }^{3}$, de leur existence et de leur fonctionnement. Après une série de remarques sur le cerveau, les viscères du bas-ventre et le rappel des conclusions de l'anatomiste Morgagni sur la consistance de cerveaux d'imbéciles et de fous, Cabanis rassemble dans une même formule les «affections hypocondriaques et mélancoliques», indiquant que pour lui le mal lié aux hypocondres et la mélancolie ne sont qu'une seule et même chose. Les maladies de l'âme doivent donc se comprendre comme des dérèglements physiques mais, il le souligne, «L'histoire des affections hypocondriaques n'a jamais été traitée dans cet esprit [...]», alors même qu'«il est facile de sentir que rien ne met plus à nu l'artifice physique de la pensée» ${ }^{4}$.

1 Lus à l'Institut en 1795 et 1796, les six premiers mémoires sont publiés en 1798/99 dans le Recueil des travaux de la classe des sciences morales et politiques, à laquelle appartenait Cabanis.

2 Cabanis 1980, 78.

3 Cabanis 1980, 89.

4 Cabanis 1980, 94. 
Ici, la question qui se pose est celle de l'étendue de la rupture proclamée avec tant de force. Après tout, indiquer qu'il y a un aspect physique de la pensée, n'est-ce pas retrouver cette tradition dont Cabanis se réclame d'ailleurs explicitement, qui va d'Hippocrate et Aristote à Bacon, Locke et Condillac? Essayer de saisir quelle peut être la rupture proposée par Cabanis, suppose donc d'examiner l'analyse qu'il propose de la mélancolie à la lumière de l'histoire dont il se veut l'héritier.

\section{La définition du tempérament mélancolique}

Le passage du premier mémoire que nous venons de citer sur l'étude des aspects physiques de l'hypocondrie pourrait laisser croire qu'une partie importante des Rapports est consacrée à cette maladie. Or il n'en est rien, et aucun mémoire ne traite exclusivement de la mélancolie. En revanche, la figure de l'hypocondriaque et les affections particulières de la région des hypocondres sont évoquées à de nombreuses reprises, bien que rapidement parfois, tout au long de l'ouvrage. La mélancolie est alors bien analysée, suivant le point de vue qui règle tout l'ouvrage: «le moral n'est que le physique». Arrêtons-nous sur l'ensemble des courtes études de la mélancolie qui parcourent les différents mémoires, et auxquelles répondent comme en miroir certains passages d'autres écrits de Cabanis, rédigés vers la même époque, tels que Discours de clôture du cours sur Hippocrate ou Coup d'œil sur les révolutions et réforme de la médecine.

Dans le sixième mémoire des Rapports, qui porte sur l'«Influence des tempéraments sur la formation des idées et des affections morales», Cabanis décrit le tempérament mélancolique, un des six tempéraments qu'il distingue, s'éloignant sur ce point des Anciens qui n'en reconnaissaient que quatre. Il considère en effet comme un de ses apports à la science médicale d'avoir signalé l'existence de deux autres tempéraments, l'un qui «est caractérisé par la prédominance du système nerveux $[\ldots]$ sur le système musculaire $[\ldots]$ », et le deuxième qui en est la version inversée, celui «qui se distingue [...] par la prédominance du système moteur sur le système sensitif ${ }^{5}$. Il diffère ici des Anciens, mais il les reprend fidèlement dans ses définitions des quatre premiers tempéraments, comme il se plaît d'ailleurs à le souligner ${ }^{6}$.Il retrouve bien en effet le sanguin, le bilieux, le flegmatique et le mélancolique, c'est-

5 Cabanis 1980, 295.

6 Cabanis 1980, 287f.: «Jusqu'ici ne dirait-on point que nous n'avons fait que suivre pas à pas la doctrine des médecins grecs, la raccorder avec les faits anatomiques, l'exposer sous un nouveau pointe vue?» 
à-dire la liste qui figure dans les écrits hippocratiques et à laquelle tous les médecins se réfèrent depuis lors.

Comme on le sait, cette liste repose sur une correspondance entre chaque tempérament et une humeur dominante; pour le sanguin, c'est le sang, pour le bilieux, c'est la bile jaune, pour le flegmatique, la pituite, et pour le mélancolique, la bile noire. On ne peut oublier, cependant, que Cabanis ajoute un complément significatif à la définition des tempéraments, puisqu'il accorde une très grande place à la physiologie, soulignant dans chaque cas l'importance prise par un organe. Si on y regarde de près, on voit que le rôle des poumons, chez le sanguin, s'accompagne d'une grande énergie du système sanguin $^{7}$, que la «prédominance du volume du foie» ${ }^{8}$, chez le bilieux, fait qu'il produit une grande quantité de bile et que, de la même manière, la faiblesse de certains organes, chez le flegmatique, permet aux «dégénérations muqueuses» de se répandre dans tout l'organisme ${ }^{9}$. Comme on le voit, Cabanis reprend, dans son approche des trois tempéraments, les humeurs des Anciens.

Dans sa description du tempérament mélancolique, en revanche, on ne trouve aucune trace de la bile noire. Physiquement, le mélancolique se distingue par «une poitrine étroite et serrée, jointe à la constriction habituelle du système épigastrique» ${ }^{10}$. L'épigastre est la région circonscrite de chaque côté par les hypocondres, ce qui justifie l'identification entre hypocondrie et mélancolie que nous avons signalée en introduction. En confondant ainsi hypocondrie et mélancolie, comme il le fait dès le premier mémoire, Cabanis suit une certaine doctrine. Le corpus hippocratique, et tout particulièrement la définition VI,23 des Aphorismes ${ }^{11}$, insiste sur les symptômes que nous appellerions aujourd'hui psychologiques et fait des sentiments prolongés de crainte et de tristesse les seuls symptômes de la mélancolie. Mais il existe une autre tradition, retracée par Jackie Pigeaud dans La maladie de l'âme, qui va de Dioclès à Pinel, en passant par Caelius Aurélien et Sydenham, et qui associe le sentiment du malheur et les douleurs de la région des hypocondres $^{12}$. Cabanis, il est vrai, ne cite pas ces auteurs de l'Antiquité, mais il avait une connaissance précise des écrits de Galien qui, lui, a commenté avec précision ces mêmes textes.

C'est Galien, d'ailleurs, comme le rappelle encore Pigeaud, qui a associé mélancolie et rétention sexuelle, assimilant mélancolie masculine et hystérie

7 Cabanis 1980, 277.

8 Cabanis 1980, 280.

9 Cabanis 1980, 295.

10 Cabanis 1980, 286.

11 Hippocrate 1994,464: «Quand la crainte et la tristesse persistent longtemps, c'est l'état mélancolique.»

12 Pigeaud 1989, 128-130. 
féminine dans un mouvement que Sydenham reprendra des siècles plus tard. Or, si la bile noire est totalement absente dans l'étiologie de la mélancolie proposée par Cabanis, une autre substance fluide apparaît comme essentielle, l'«humeur séminale»:

Chez le mélancolique, c'est l'humeur séminale elle seule qui communique une âme nouvelle aux impressions, aux déterminations, aux mouvements; c'est elle qui crée, dans le sein de l'organe cérébral, ces forces étonnantes, trop souvent employées à poursuivre des fantômes, à systématiser des visions. ${ }^{13}$

Ce passage est remarquable car Cabanis y propose à la fois une définition et une étiologie de la mélancolie. Cette maladie se caractérise par un certain type de désordre de la pensée, le délire, et par une surabondance de l'«humeur séminale», un dysfonctionnement de la sexualité. Un lien est donc établi entre le moral et le physique, mais le sexuel semble tout à coup prendre la place que Cabanis paraissait jusqu'ici vouloir attribuer aux hypocondres. La mélancolie a-t-elle deux origines physiques? Quel est le rôle des hypocondres? La réponse à ces questions ne se trouve pas dans le mémoire sur les tempéraments, mais dans celui qui traite de «l'influence des sexes sur le caractère des idées». Cabanis y décrit la place des hypocondres et des organes sexuels dans le réseau nerveux qui court dans l'ensemble du corps humain. Il indique d'abord:

La nature semble avoir à dessein placé les ganglions et les plexus dans le voisinage des viscères, où l'influence nerveuse doit être le plus considérable. L'épigastre et la région hypocondriaque en sont comme tapissés: aussi leur sensibilité est-elle extrêmement vive, leur sympathie extrêmement étendue $[\ldots] \cdot{ }^{14}$

Et il ajoute:

[...] les nerfs des parties de la génération, dans l'un et dans l'autre sexe [...] sont formés de beaucoup de nerfs différents: ils ont des relations avec ceux de tous les viscères du bas-ventre $[\ldots] .^{15}$

Une réciprocité se dessine dans laquelle la densité du réseau nerveux des hypocondres prolonge et amplifie les sensations éprouvées par le bas-ventre, tout comme les organes sexuels perçoivent mieux que d'autres lieux du corps les excitations qui atteignent la région des hypocondres.

Malgré la relation établie entre le ventre et le bas-ventre, c'est pourtant davantage ce dernier, plus précisément les désordres qui l'affectent, que Cabanis désigne comme origine physique de la mélancolie:

Les organes de la génération [...] sont très souvent le siège véritable de la folie. Leur sensibilité vive est susceptible des plus grands désordres: l'étendue de leur influence sur tout le système fait que ces désordres deviennent presque tous généraux, et sont principalement ressentis par le centre cérébral. ${ }^{16}$

13 Cabanis 1980, 287.

14 Cabanis 1980, 225.

15 Cabanis 1980, 225.

16 Cabanis 1980,115f. 
Le terme de folie utilisé ici ne désigne pas l'ensemble des désordres psychologiques dont la mélancolie ne serait qu'une des formes possibles. Il est bien davantage le synonyme de la seule mélancolie, comme le montre la suite où Cabanis analyse la «mélancolie amoureuse» et la «mélancolie ascétique» pour illustrer sa théorie ${ }^{17}$. De cette manière, il fait se rejoindre la tradition issue de Galien qui insiste sur une étiologie sexuelle et un autre texte essentiel dans l'histoire de la mélancolie, le célèbre Problème $X X X$ qui met en avant le délire et l'obsession pour le sexe parmi les caractères principaux de la mélancolie et fait le lien avec les délires prophétiques, ou ce que l'on peut également appeler «enthousiasme» ${ }^{18}$. Juste avant d'affirmer l'importance de l'«humeur séminale», Cabanis souligne que les formes exaltées de croyance ne sont que des masques de la mélancolie. Le bon observateur la reconnaît, dit-il, dans

les austérités d'une morale excessive, dans les extases de la superstition, dans ces maladies extraordinaires qui jadis constituaient certains individus de l'un et l'autre sexe prophètes, augures ou pythonisses $[\ldots] \cdot{ }^{19}$

La fin de ce passage évoque inévitablement le Problème $X X X$ où «les Sibylles, les Bacis, et tous ceux qui sont inspirés» apparaissent comme autant de figures du mal de la bile noire. Il reprend aussi le thème de la mélancolie religieuse en insistant sur le délire, de telle manière que la mélancolie semble moins se caractériser pour Cabanis par la souffrance et la tristesse que par l'inadéquation entre la pensée et le réel.

\section{Les circonstances et le cerveau}

Mettre l'accent sur le délire amène nécessairement à s'interroger sur son origine. Comment expliquer cette perception erronée du réel? Qu'est-ce qui a pu la provoquer? Cabanis se livre à plusieurs reprises, tout au long de son œuvre, à l'étude minutieuse des différents cas où le délire, l'imagination exaltée sont associés à la mélancolie. Ainsi il reprend, dans les Rapports, ces histoires bien connues de la littérature de la mélancolie où l'on voit les «hypocondriaques» en proie à des «illusions» et des «visions [...] ridicules», croyant «avoir des jambes de verre ou de paille, ou n'avoir point de tête, ou qui soutenaient que leur corps renfermait d'immenses amas d'eau $[\ldots] \gg^{20}$.

17 Cabanis 1980, 116.

18 Aristote 1988, 97: «Mais beaucoup [de ceux qui sont atteints du mal de la bile noire] sont saisis des maladies de la folie ou de l'enthousiasme. Ce qui explique les Sibylles, les Bacis, et tous ceux qui sont inspirés [...].»

19 Cabanis 1980, 287.

20 Cabanis 1980, 149. 
D'une manière remarquable, il les lie aux crises d'exaltation religieuse traversées par le naturaliste hollandais Swammerdam, dont l'exemple vient illustrer le cas général des hypocondriaques, victimes de «visions ridicules», mais qui «étaient capables, pendant ce temps, d'exécuter des travaux fort ingénieux ${ }^{21}$. Le choix de Swammerdam n'est pas fortuit, car ce savant vient rejoindre le grand ensemble des personnes chez qui les facultés nécessaires au raisonnement sont très développées et dont Cabanis souligne, à maintes reprises, la fragilité. Un groupe est plus susceptible que d'autres de souffrir de la mélancolie, c'est celui constitué par «les hommes livrés aux affaires, des savants, des gens de lettres, des artistes, et [...] toutes les personnes dont la vie n'est pas dévouée à des travaux simplement manuels» ${ }^{22}$. Une activité intellectuelle très grande, supérieure à la normale les caractérise.

Or, suivant le principe de l'identité du physique et du moral, cette «existence morale» a un lieu dans le corps humain, un pendant physique, un organe. Les Rapports l'affirment d'emblée, cet organe, c'est le cerveau. Dans une formule frappante, Cabanis indique dans le premier mémoire que le cerveau produit la pensée, «de même que l'estomac et les intestins [opèrent] la digestion du foie» ${ }^{23}$. Il soutient ainsi une position matérialiste radicale suivant laquelle les sensations convergent toutes vers un même centre, le cerveau, qui les transforme et les redistribue. On comprend alors que toute analyse des délires de l'imagination amène Cabanis à s'interroger sur le fonctionnement du cerveau. Il attribue à ce dernier autonomie et activité, s'opposant ainsi à la conviction alors largement partagée suivant laquelle le cerveau serait une masse insensible et inerte ${ }^{24}$. Il est important de noter qu'il ne se livre ni dans les Rapports ni dans Révolutions et réforme de la médecine, ouvrage pourtant à portée historique, à une récapitulation des avancées majeures des anatomistes dans l'étude du cerveau depuis la fin du $\mathrm{XVII}^{\mathrm{e}}$ siècle. Cette question est laissée de côté dans Révolutions et réforme, et les Rapports privilégient les travaux de Morgagni sur ceux des autres savants qui ont mené des recherches tout aussi importantes sur le cerveau. L'italien Morgagni a en effet l'avantage sur le britannique Thomas Willis ou sur son compatriote Baglivi (pour ne citer que les plus célèbres de ces anatomistes) de s'être expressément intéressé aux cerveaux des fous et d'y avoir découvert la trace physique de l'affection qui les tourmentait. Dans ses dissections, il reconnaît une «inégalité de consistance dans la pulpe du cerveau» des fous ${ }^{25}$.

21 Cabanis 1980, 149.

22 Cabanis 1823, 349.

23 Cabanis 1980, 137.

24 Voir, par exemple, article «cerveau» dans Encyclopédie, tome II.

25 Cabanis 1980, 93. 
Pour Cabanis, il s'agit encore et toujours d'affirmer une identité sans failles entre le physique et le moral. Un débat se devine en filigrane chaque fois qu'il est question de Morgagni dans les Rapports. Dans le premier mémoire, tout comme dans le dixième, il fait état de la position de Pinel, qui diverge de la sienne. Pinel est alors, dans la configuration nouvelle du savoir soutenue par Cabanis, Destutt de Tracy et leurs alliés, l'homme des maladies de l'âme, celui qui doit établir la théorie moderne des affections du moral. Or, cela est patent dans le Traité philosophique sur l'aliénation mentale, il est très difficile pour Pinel d'adhérer aux différentes «découvertes» qui posent que toute maladie mentale a une traduction dans le physique, que celle-ci soit une anormalité dans la configuration du cerveau ou une configuration particulière de la tête. A chaque fois qu'il le mentionne, Cabanis fait tout pour atténuer l'opinion de Pinel, rapportée en note de bas de page, ou présentée comme ambiguë ${ }^{26}$.

Les malformations du cerveau lui permettent ainsi d'expliquer des dysfonctionnements moraux ou des formes d'intelligence hors normes. Il établit ainsi, en s'appuyant sur les travaux de Morgagni, un lien direct entre «le défaut d'harmonie des fonctions (intellectuelles)» et l'«augmentation, diminution, ou plus souvent grande inégalité de consistance du cerveau» ${ }^{27}$. Pour Cabanis, suivant les découvertes des anatomistes du XVII ${ }^{\mathrm{e}}$ siècle, le cerveau est constitué de nerfs, répartis dans différentes «couches», tous en relation les uns avec les autres et avec l'ensemble du système nerveux, qu'ils commandent. Mais, à la différence de la plupart de ses contemporains, il considère le cerveau comme un organe actif et sensible. Cette sensibilité du cerveau est au cœur de l'étiologie nouvelle de la mélancolie qu'il propose.

Un passage de Révolution et réforme, déjà cité, éclaire la relation qu'il établit entre le genre particulier de sensations ressenties par les mélancoliques et les nerfs du cerveau. Se penchant sur ces «personnes dont l'existence morale a pris son entier développement ${ }^{28}$, Cabanis s'exclame:

Que de fibres peuvent être ébranlées par les plus faibles causes, quand la tête reçoit et combine beaucoup d'impressions; quand beaucoup de sentiments fermentent dans le cœur! ${ }^{29}$

26 Cabanis 1980, 93, où la note 1 fait état d'une opposition possible, mais vite contrée: «Pinel n'a souvent rien montré de semblable, mais les faits recueillis par Morgagni et par quelques autres doivent être regardés comme certains [...].» Page 558, la même rhétorique est à l'œuvre: «Pinel affirme n'avoir rien découvert de semblable [...] et l'on peut compter entièrement sur les assertions d'un observateur si sagace et si scrupuleusement exact; mais il est impossible aussi de rejeter celles de plusieurs savants anatomistes, non moins dignes de foi.»

27 Cabanis 1980, 559.

28 Cabanis 1823, 349.

29 Cabanis 1823, 349. 
Un déséquilibre apparaît ici entre le volume important des sensations éprouvées par les mélancoliques et la «faiblesse» de ce qui les suscite. Cette dysharmonie correspond à celle découverte par les anatomistes: la sensibilité excessive et désordonnée du mélancolique s'explique par les différences de consistance observées dans le cerveau. Pour Cabanis, ce qui caractérise l'hypocondrie, c'est l'incapacité du cerveau d'analyser correctement les sensations qui lui sont transmises, créant ainsi ces distorsions par rapport au réel qui expliquent la grande imagination des artistes et des savants tous comme les délires des «sibylles» et des prophètes.

La place qu'il accorde au cerveau permet à Cabanis d'élaborer une étiologie nouvelle de la mélancolie qui correspond à son principe de l'identité du physique et du moral, tout en reprenant l'essentiel de ce que la tradition médicale lui a transmis sur cette affection morale. Grâce à cette analyse, il peut également intégrer dans sa conception de la mélancolie la sexualité exacerbée, signalée dans le Problème $X X X$ comme un caractère majeur de l'hypocondrie, ainsi que l'influence de circonstances extérieures, telles que le climat et l'âge, auxquelles le corpus hippocratique accorde une grande importance. En effet, Cabanis a posé que la région du bas-ventre et le cerveau sont reliés par un réseau nerveux particulièrement dense. Ce qui affecte l'un affecte forcément l'autre. La suractivité cérébrale n'est donc que l'autre face d'une suractivité sexuelle, que celle-ci soit apparente ou que le mélancolique fasse tout pour la cacher ou l'ignorer.

Le Problème $X X X$ qui, dès l'attaque, indique qu'il va être question des «hommes d'exception, en ce qui regarde la philosophie, la science de l'Etat, la poésie ou les arts», affirme par ailleurs clairement que «les mélancoliques, pour la plupart, sont obsédés par le sexe» ${ }^{30}$. De la même manière, dans son analyse de la mélancolie de l'habitant des pays chauds, Cabanis reprend les remarques des traités hippocratiques Airs, eaux, lieux et De la nature de l'homme qui associent tempérament mélancolique et situation géographique, recrudescence de la bile noire et saison des pluies. Il remarque ainsi que «Les pays qui fournissent une nourriture facile, surtout quand la chaleur y vient augmenter le penchant à l'oisiveté qu'inspire l'abondance, ces pays énervent les forces corporelles». ${ }^{31}$ Plus loin, il décrit les conséquences de façon plus précise:

Les maladies hypocondriaques les plus sombres, les penchants les plus bizarres et les plus égarés $[\ldots]$ en sont fréquemment la suite fatale $[\ldots] .32$

30 Aristote 1988, 91.

31 Cabanis 1980, 457

32 Cabanis 1980, 459. 
Dans ces pays où tout ce qui est nécessaire à la vie est fourni à l'homme en abondance, sans qu'il ait à fournir d'efforts pénibles, où il n'a pas à lutter contre les éléments pour assurer sa subsistance, la mélancolie trouve le terrain le plus accueillant. Cette situation étonnante a une explication: les climats chauds hâtent l'explosion de la puberté;les organes sexuels atteignent leur plein développement alors que le reste du corps demeure, lui, à un stade antérieur. C'est ainsi que:

De cette excitation précoce qui agit particulièrement sur certains organes et sur certaines fonctions, ou plutôt de ce défaut d'équilibre entre les diverses parties du système vivant, s'ensuivent des modifications singulières de toute l'existence morale. ${ }^{33}$

La mélancolie est clairement liée ici à cette «influence particulière des organes de la génération $»^{34}$, signalée dans le mémoire sur les tempéraments, et cette influence, on le voit, est celle qui est due à une domination du sexuel produite par les circonstances.

\section{Le rôle de la sensibilité}

Quelle que soit son origine, cependant, la mélancolie est avant tout la conséquence d'une dysharmonie. Chez l'homme de génie ou d'imagination, c'est l'activité cérébrale qui prend le pas sur toutes les autres, chez l'habitant des pays chauds, les organes sexuels se développent indépendamment de l'ensemble du corps. De la même manière, la mélancolie de l'homme mûr, que les Rapports analysent attentivement, trouve sa source dans un système circulatoire engorgé qui ralentit les mouvements vitaux. Les «passions ambitieuses, tristes et personnelles ${ }^{35}$ qui caractérisent cet âge, d'après Cabanis, naissent d'un affaiblissement qui touche en particulier «les parties flottantes du bas-ventre [dont] les viscères [...] sont sujets à s'obstruer» ${ }^{36}$. On cherche alors à compenser le sentiment de la diminution des forces par une imagination «qui a dès lors besoin de se rassurer par les impressions d'une force factice, exercée sur des objets extérieurs $»^{37}$. Le délire mélancolique s'installe.

L'hypocondrie se caractérise donc toujours par la domination de certains organes sur les autres et par une masse exceptionnelle d'idées en grande partie inadéquates. L'homme de génie est aussi celui qui se trompe tout en découvrant une vérité jusqu'alors cachée. Swammerdam illustre parfaitement ce paradoxe:

33 Cabanis 1980, 348.

34 Cabanis 1980, 287.

35 Cabanis 1980, 215.

36 Cabanis 1980, 207.

37 Cabanis 1980, 206. 
C'est au milieu des accès de la plus terrible hypocondriasie que Swammerdam faisait ses plus brillantes recherches. Mais s'étant mis dans la tête que Dieu pouvait s'offenser d'un examen si curieux de ses œuvres, il commença par renoncer à poursuivre de très belles expériences sur les injections [...] et dans un paroxysme plus violent, il finit par livrer aux flammes une grande partie de ses manuscrits. ${ }^{38}$

Ce passage souligne la violence à l'œuvre dans l'imagination du mélancolique. Cette violence se retrouve dans tous les cas décrits par Cabanis où l'idée d'excès est constamment présente. Que ce soit dans les «âmes vives et ardentes ${ }^{39}$, qu'il trouve dans les pays chauds, ou plus généralement dans «la grande énergie et l'activité constante du cerveau $»^{40}$, qui d'après lui caractérise tous les mélancoliques, Cabanis analyse toujours cette affection suivant les lois de la mécanique. La mélancolie est le résultat d'une force exceptionnelle.

Mais de quelle force est-il question? Quelle est la nature de cette masse d'énergie que l'observateur découvre à l'œuvre dans le cerveau ou les hypocondres? Il s'agit, nous le savons, de la force qui est à l'origine des sensations et des idées. Pour Cabanis, héritier du sensualisme de Condillac et de la philosophie de la connaissance de Locke $^{41}$, toutes les impressions et toutes les idées sont le fait de la sensibilité. Il l'affirme à maintes reprises, «la sensibilité physique est la source de toutes les idées et de toutes les habitudes qui constituent l'existence morale de l'homme» ${ }^{42}$.

Dans son analyse de la mélancolie, la sensibilité est bien une propriété des organes, mais elle permet à chacun d'établir une relation avec le monde extérieur et avec lui-même. Elle possède également une étonnante autonomie. Loin d'être une qualité constante de chaque organe, elle varie en intensité suivant les circonstances, climat, âge ou occupation. C'est cette variation et les lieux qu'elle privilégie dans le corps qui provoquent la mélancolie.

Cabanis défend une conception très nouvelle de la sensibilité qui lui permet, cependant, de rester fidèle à la tradition issue de l'Antiquité, tout en prenant en compte les dernières découvertes de l'anatomie, et en intégrant la philosophie sensualiste. Il explique les sensations plus ou moins fortes, plus ou moins fines, en assimilant la sensibilité à une masse, une masse d'énergie qui se déplace. Dans les Rapports, il décrit le fonctionnement de la sensibilité à l'aide d'une image particulièrement éclairante:

38 Cabanis 1980, 149.

39 Cabanis 1980, 349.

40 Cabanis 1980, 287.

41 Cabanis est toujours très précis sur cette filiation. Voir en particulier, dans les Rapports, la préface et le premier Mémoire.

42 Cabanis 1980, 103. 
Remarquons [...] que la sensibilité se comporte à la manière d'un fluide, dont la quantité totale est déterminée, et qui, toutes les fois qu'il se jette en plus grande abondance dans un de ses canaux, diminue proportionnellement dans les autres. ${ }^{43}$

A l'époque où ce texte est rédigé, la comparaison entre la sensibilité et «un fluide» renvoie nécessairement à Mesmer, dont le nom est d'ailleurs évoqué quelques lignes plus loin. Comme tout savant respectable, Cabanis condamne Mesmer dont les pratiques étaient celles, dit-il, d'un charlatan. Mais il ne faut pas oublier que cette critique est le fait d'un ancien disciple qui doit marquer ses distances ${ }^{44}$. Cabanis sent bien qu'à utiliser une telle image, il risque d'être rangé dans le camp des mesméristes. Si l'on ne peut douter de l'éloignement, d'ailleurs rapide, de Cabanis du cercle de Mesmer, il n'en reste pas moins qu'il semble avoir trouvé là une manière d'envisager la sensibilité particulièrement utile. La comparaison entre fluide et sensibilité lui permet en effet de rendre compte de la relation entre masse et harmonie. Elle indique que l'abondance ne peut être que l'autre nom de l'excès et qu'elle entraîne nécessairement un déséquilibre. A une sensibilité «vive» ici, répondra nécessairement un manque là, c'est-à-dire des organes tronqués puisque d'autres auront été privilégiés à leur dépens.

La sensibilité apparaît ainsi comme un volume constant d'énergie, qui se déplace suivant les circonstances. La relation que Cabanis établit entre la masse du fluide et sa force révèle une importante influence de la mécanique newtonienne et, en particulier, de la deuxième loi, communément appelée de l'action-réaction. Pour expliquer l'étroite interdépendance entre le cerveau et les organes de la génération, il associe ce schéma hérité de Newton aux connaissances anatomiques. La grande activité de l'organe cérébral suppose la réception, par cet organe, d'un grand nombre de sensations et la production d'un volume considérable d'idées. Il transforme les sensations en idées suivant un schéma en deux temps qui est celui de l'action-réaction. Il y a en effet d'abord perception, puis reflux, de sorte que «pour tout dire en un mot, les nerfs exercent sur eux-mêmes une véritable réaction pour le sentiment» ${ }^{45}$.

Or, à faire des idées et des sentiments (qui sont une forme d'idée) des sensations transformées et de la répartition de la sensibilité le produit des circonstances, Cabanis non seulement propose une étiologie originale de la mélancolie, mais ouvre également la porte à de nouvelles formes de cures. Un passage de Quelques principes et quelques vues sur les secours publics, dans lequel il est question des «maisons publiques et charitables de fous», décrit cet avenir utopique:

43 Cabanis 1980, 134.

44 Voir Vinchon 1999.

45 Cabanis 1980, 134. 
J'oserai ajouter que, par l'effet des institutions sages qui constituent une véritable république, la démence et tous les désordres de l'esprit, doivent également devenir plus rares. [...] Soumis aux seules douleurs qui sont inséparables de sa nature, [l'homme] ignorera toutes les altérations de l'esprit, que produisent directement les désordres d'un mauvais état social, et par suite, les funestes penchants que développe son influence. Enfin, le moment viendra peut-être, où la folie n'aura d'autre source que le dérangement primitif de l'organisation, ou ces accidents singuliers de la vie humaine, qu'aucune sagesse ne peut prévenir. ${ }^{46}$

Puisque la sensibilité est à la fois sous l'influence des circonstances extérieures (le climat, le métier, par exemple) et intérieures (les modifications de certains organes du fait de l'âge, par exemple), Cabanis est amené à distinguer entre une folie «de nature» due à une disposition originelle des organes et celle qui est le produit de l'extérieur, c'est-à-dire de la société. Nous ne traiterons pas ici de la manière dont Cabanis analyse les inégalités sociales et leur influence néfaste sur la santé de chacun. Remarquons seulement que, au terme de notre étude, il apparaît que c'est l'ensemble des circonstances de la vie qui doivent être modifiées pour assurer le bien-être physique et moral de tous.

Le XVIII ${ }^{e}$ siècle se clôt donc sur une dernière métamorphose de la mélancolie. La tradition médicale issue de l'Antiquité - qui connaît alors une véritable «Renaissance», comme Pigeaud l'a maintes fois signalée ${ }^{47}$ - rejoint les dernières données de l'anatomie pathologique. L'analyse que fait Cabanis de l'affection mélancolique est pourtant radicalement originale:il conçoit ce mal à la fois comme une affection du moral (la très grande tristesse, l'imagination exaltée), et comme un dysfonctionnement physique qui concerne certains organes (en l'occurrence, le cerveau et le bas-ventre). Alors que Pinel ne veut s'en tenir qu'à l'aspect moral de la mélancolie, l'insistance sur le pendant physique de celle-ci permet à Cabanis de défendre le caractère réversible du mal. Pinel est obligé de reconnaître que les rémissions de ses patients ont souvent été partielles, et de courte durée. Faire de la sensibilité un fluide et penser l'influence des circonstances comme autant de chocs subit par le corps ouvre la voie à la guérison. Freud et Breuer s'en souviendront en rédigeant leurs Etudes sur l'Hystérie ${ }^{48}$ : ils expliqueront en effet le sommeil avec rêves et le sommeil sans rêves à partir de la masse d'énergie présente dans le corps du dormeur et de sa répartition.

46 Cabanis 1989 [1803], 527f.

47 Pigeaud 2000.

48 Freud/Breuer 2005, 155, n. 1. 


\section{Bibliographie}

Aristote, L’homme de génie et la mélancolie, Problème XXX,1, traduction, présentation et notes par Jackie Pigeaud (Paris 1988)

Cabanis, Pierre Jean Georges, Euvres complètes, Tome I (Paris 1823)

- Rapports du physique et du moral de l'homme (Paris et Genève 1980 [reprint de l'édition de 1844])

- Du Degré de certitude de la médecine (Paris et Genève 1989 [reprint de l'édition de 1803])

Freud, Sigmund/Joseph Breuer, Etudes sur l'hystérie (Paris 2005)

Hippocrate, De l'art médical, traduction d'Emile Littré, présentation, commentaires et notes par Danielle Gourevitch (Paris 1994)

Pigeaud, Jackie, La maladie de l'âme (Paris 1989)

- Aux portes de la psychiatrie. Pinel, l'Ancien et le Moderne (Paris 2000)

Vinchon, Jean, Mesmer et son secret (Paris et Montréal 1999) 